\title{
THE INTERPOLATION PROOF OF GROTHENDIECK'S INEQUALITY
}

\author{
by G. J. O. JAMESON
}

(Received 22nd June 1984)

\section{Introduction}

This note is an exposition of the simple and elegant approach to Grothendieck's inequality given in [2] and [4], with one further simplification. The process of factorizing through $L_{2}([2]$, p. 21) introduces a factor of $\sqrt{\pi / 2}$ into the final constant. We show that this step can be avoided.

The ingredients are Khinchin's inequality, an interpolation result for $p$-summing norms and a reformulation of Grothendieck's inequality. No measure theory is used at any stage. We obtain Grothendieck's inequality with constant $2 \sqrt{3}$. We present the details for the real case, but the method applies with minor changes to the complex case too (giving the same constant). Some short proofs of known results are included for completeness.

\section{Preliminaries}

If $X, Y$ are normed linear spaces, we denote by $U_{X}$ the unit ball in $X$ and by $L(X, Y)$ the space of continuous linear operators from $X$ to $Y$.

We denote by $l_{p}^{n}$ the space $\mathbb{R}^{n}$ with $l_{p}$-norm. The $i$ th unit vector is denoted by $e_{i}$, and the usual inner product on $\mathbb{R}^{n}$ by $\langle$,$\rangle .$

Given a finite sequence $\left(x_{1}, \ldots, x_{k}\right)$ of elements of a normed linear space, define $\mu_{p}\left(x_{1}, \ldots, x_{k}\right)$ (for $p \geqq 1$ ) by

$$
\left[\mu_{p}\left(x_{1}, \ldots, x_{k}\right)\right]^{p}=\sup \left\{\sum_{i}\left|f\left(x_{i}\right)\right|^{p}: f \in U_{X^{*}}\right\} .
$$

It is elementary that if $K$ is a norming subset of $U_{X^{*}}$, then this is equal to

$$
\sup \left\{\sum_{i}\left|f\left(x_{i}\right)\right|^{p}: f \in K\right\}
$$

In particular, if $X$ is $l_{\infty}^{n}$ or $C(S)$, then (taking $K$ to be the set of point-evaluations) we 
have

$$
\left[\mu_{p}\left(x_{1}, \ldots, x_{k}\right)\right]^{p}=\left\|\sum_{i}\left|x_{i}\right|^{p}\right\|
$$

where $p$ th powers are defined in the obvious pointwise sense.

The $p$-summing norm of an operator $T$ is defined by

$$
\pi_{p}(T)=\sup \left\{\left(\sum_{i}\left\|T x_{i}\right\|^{p}\right)^{1 / p}: \mu_{p}\left(x_{1}, \ldots, x_{k}\right) \leqq 1\right\},
$$

where finite sequences of any length $k$ are considered.

One of the equivalent forms of Grothendieck's inequality is; there is a constant $K_{G}$ such that for any $m, n$ and any $T$ in $L\left(l_{1}^{m}, l_{2}^{n}\right)$, we have $\pi_{1}(T) \leqq K_{G}\|T\|$. The best possible value of $K_{G}$ (which is still unknown) is called "Grothendieck's constant".

Let $D_{k}$ denote the set of all mappings $\varepsilon$ from $\{1, \ldots, k\}$ to $\{-1,1\}$. Khinchin's inequality states that for $a=\left(a_{1}, \ldots, a_{k}\right) \in \mathbb{R}^{k}$,

$$
2^{-k} \sum_{\varepsilon \in D_{k}}|\langle\varepsilon, a\rangle| \geqq \frac{1}{\sqrt{2}}\left(\sum_{i} a_{i}^{2}\right)^{1 / 2} .
$$

This has long been known, and is easily proved, with $1 / \sqrt{3}$ instead of $1 / \sqrt{2}$. For a proof that the best constant is $1 / \sqrt{2}$, see [1].

The quantity $\tilde{\pi}_{2}(T)$

Let $Y$ be $\mathbb{R}^{n}$ with any norm under which it is a Banach lattice (in particular, any $l_{p}^{n}$ ). For $y_{1}, \ldots, y_{k}$ in $Y$, the element $\left(y_{1}^{2}+\ldots+y_{k}^{2}\right)^{1 / 2}$ is well-defined. For $T$ in $L\left(l_{\infty}^{m}, Y\right)$, define

$$
\tilde{\pi}_{2}(T)=\sup \left\{\left\|\left[\sum_{i}\left(T a_{i}\right)^{2}\right]^{1 / 2}\right\|: \mu_{2}\left(a_{1}, \ldots, a_{k}\right) \leqq 1\right\} .
$$

One can verify that $\tilde{\pi}_{2}$ is a norm, though this is not important for our purposes. Its relevance here is that it provides an equivalent statement of Grothendieck's inequality, as follows.

Proposition 1. $K_{G}=\sup \left\{\tilde{\pi}_{2}(T): T \in L\left(l_{\infty}^{m}, l_{1}^{P}\right),\|T\| \leqq 1, m, p \in \mathbb{N}\right\}$.

Proof. Any mapping $S$ in $L\left(l_{1}^{m}, l_{2}^{n}\right)$ can be written as

$$
S x=\sum_{i=1}^{n}\left\langle a_{i}, x\right\rangle e_{i}
$$

where $a_{i} \in l_{\infty}^{m}$. Then $\|S x\|^{2}=\sum_{i}\left\langle a_{i}, x\right\rangle^{2}$, so $\|S\|=\mu_{2}\left(a_{1}, \ldots, a_{n}\right)$. We consider $S$ with $\|S\|=1$. 
Any mapping $T$ in $L\left(l_{\infty}^{m}, l_{1}^{p}\right)$ can be written as

$$
T y=\sum_{j=1}^{p}\left\langle b_{j}, y\right\rangle e_{j}
$$

where $b_{j} \in l_{1}^{m}$. Then $\|T\|=\mu_{1}\left(b_{1}, \ldots, b_{p}\right)$. We consider $T$ with $\|T\|=1$.

With $S, T$ as above, we have $\left(T a_{i}\right)(j)=\left\langle a_{i}, b_{j}\right\rangle$, so if

$$
c=\left[\sum_{i}\left(T a_{i}\right)^{2}\right]^{1 / 2},
$$

then

$$
c(j)^{2}=\sum_{i}\left\langle a_{i}, b_{j}\right\rangle^{2}=\left\|S b_{j}\right\|^{2},
$$

so $\|c\|=\sum_{j}\left\|S b_{j}\right\|$ (where the norms are those of the appropriate spaces). Hence the statement that $\tilde{\pi}_{2}(T) \leqq K$ for all such $T$ is equivalent to the statement that $\pi_{1}(S) \leqq K$ for all such $S$.

Let $a_{1}, \ldots, a_{k} \in \mathbb{R}^{n}$, and define $c$ by: $c \geqq 0$ and $c^{2}=\sum_{i} a_{i}^{2}$. In $l_{2}^{n}$ we have clearly $\|c\|^{2}=$ $\sum\left\|a_{i}\right\|^{2}$, and hence for $T$ in $L\left(l_{\infty}^{m}, l_{2}^{n}\right)$ we have $\tilde{\pi}_{2}(T)=\pi_{2}(T)$. The space $l_{1}^{n}$ has the following well-known property ("2-concavity").

Lemma 1. In $l_{1}^{n}$, we have $\|c\|^{2} \geqq \sum_{i}\left\|a_{i}\right\|^{2}$.

Proof. Write $\left\|a_{i}\right\|=\lambda_{i}$. By Schwarz's inequality,

$$
0 \leqq \sum_{i} \lambda_{i}\left|a_{i}\right| \leqq\left(\sum_{i} \lambda_{i}^{2}\right)^{1 / 2} c
$$

in the natural ordering of $l_{1}^{n}$. Since the norm of $l_{1}^{n}$ is additive for positive elements, it follows that

$$
\sum_{i} \lambda_{i}^{2} \leqq\left(\sum_{i} \lambda_{i}^{2}\right)^{1 / 2}\|c\|
$$

which gives the result.

Hence for $T$ in $L\left(l_{\infty}^{m}, l_{1}^{n}\right)$, we have $\tilde{\pi}_{2}(T) \geqq \pi_{2}(T)$.

The following lemma (cf. [4], Lemma 1.1) is well-known as part of the proof of the weaker version of Khinchin's inequality.

Lemma 2. Given $a_{1}, \ldots, a_{k} \in \mathbb{R}^{n}$ and $\varepsilon \in D_{k}$, write $b_{\varepsilon}=\sum_{i} \varepsilon_{i} a_{i}$. Then

$$
2^{-k} \sum_{\varepsilon \in D_{k}} b_{\varepsilon}^{4} \leqq 3\left(\sum_{i} a_{i}^{2}\right)^{2}
$$


Proof. $b_{\varepsilon}^{4}$ is the sum of terms of the form $\varepsilon_{i} \varepsilon_{j} \varepsilon_{k} \varepsilon_{l} a_{i} a_{j} a_{k} a_{l}$. When we sum over $\varepsilon \in D_{k}$, the only terms that do not cancel are

$$
\sum_{i} a_{i}^{4}+6 \sum_{i<j} a_{i}^{2} a_{j}^{2}
$$

occurring for each $\varepsilon$. The statement follows.

Recall that for non-negative numbers $c_{1}, \ldots, c_{N}$,

$$
\frac{1}{N}\left(c_{1}+\ldots+c_{N}\right) \leqq\left[\frac{1}{N}\left(c_{1}^{4}+\ldots+c_{N}^{4}\right)\right]^{1 / 4} .
$$

Proposition 2. Let $Y$ be $\mathbb{R}^{n}$ with any norm under which it is a Banach lattice. For $T$ in $L\left(l_{\infty}^{m}, Y\right)$, we have

$$
\tilde{\pi}_{2}(T) \leqq 3^{1 / 4} 2^{1 / 2} \pi_{4}(T)
$$

Proof. Take elements $a_{1}, \ldots, a_{k}$ of $l_{\infty}^{m}$ and define $b_{\varepsilon}$ as in Lemma 2. By Khinchin's inequality,

$$
\left(\sum_{i}\left(T a_{i}\right)^{2}\right)^{1 / 2} \leqq \sqrt{2} 2^{-k} \sum_{e \in D_{k}}\left|T b_{\varepsilon}\right|
$$

Since the norm in $Y$ is such that $0 \leqq u \leqq v$ implies $\|u\| \leqq\|v\|$, we have

$$
\begin{aligned}
\left\|\left(\sum_{i}\left(T a_{i}\right)^{2}\right)^{1 / 2}\right\| & \leqq \sqrt{2} 2^{-k} \sum_{\varepsilon}\left\|T b_{\varepsilon}\right\| \\
& \leqq \sqrt{2}\left[2^{-k} \sum_{\varepsilon}\left\|T b_{\varepsilon}\right\|^{4}\right]^{1 / 4} \quad \text { by the remark above } \\
& \leqq \sqrt{2} \pi_{4}(T)\left\|\left(2^{-k} \sum_{\varepsilon} b_{\varepsilon}^{4}\right)^{1 / 4}\right\| \\
& \leqq \sqrt{2} \pi_{4}(T) 3^{1 / 4}\left\|\left(\sum_{i} a_{i}^{2}\right)^{1 / 2}\right\| \quad \text { by Lemma } 2 .
\end{aligned}
$$

Since $\left\|\left(\sum_{i} a_{i}^{2}\right)^{1 / 2}\right\|=\mu_{2}\left(a_{1}, \ldots, a_{k}\right)$, this completes the proof.

\section{Interpolation}

The required interpolation result can be related to the Riesz-Thorin theorem, but it is simpler to prove it directly from Pietsch's theorem, as follows. For $T$ in $L\left(l_{\infty}^{n}, Y\right.$ ) (any 
Y), Pietsch's theorem ([3], p. 64-5) asserts the equivalence of the following statements:

(i) $\pi_{p}(T) \leqq A$,

(ii) there is a positive linear functional $\phi$ on $l_{\infty}^{n}$ such that $\|\phi\|=A^{p}$ and $\|T x\|^{p} \leqq \phi\left(|x|^{p}\right)$ for all $x$ in $l_{\infty}^{n}$.

Statement (ii) can clearly be reformulated as follows:

(ii') there exist non-negative numbers $\lambda_{1}, \ldots, \lambda_{n}$ such that $\sum_{j} \lambda_{j}=A^{p}$ and

$$
\left\|\sum_{j} x_{j} a_{j}\right\|^{p} \leqq \sum_{j} \lambda_{j}\left|x_{j}\right|^{p}
$$

for all $x=\left(x_{1}, \ldots, x_{n}\right)$ in $\mathbb{R}^{n}$, where $T e_{j}=a_{j}$.

Proposition 3. Let $1<p<r$. Then for any $T$ in $L\left(l_{\infty}^{n}, Y\right)$,

$$
\pi_{r}(T)^{r} \leqq \pi_{p}(T)^{p}\|T\|^{r-p}
$$

In particular,

$$
\pi_{2 p}(T)^{2} \leqq \pi_{p}(T)\|T\|
$$

Proof. Write $\theta=p / r$. There exist $\lambda_{j}$ as in (ii'), with $A=\pi_{p}(T)$. Take $x$ in $l_{\infty}^{n}$ and $f$ in $U_{Y^{*}}$ Write $\left|x_{j}\right|=y_{j}$ and $\left|f\left(a_{j}\right)\right|=u_{j}$. Clearly, $\sum_{j} u_{j} \leqq\|T\|$. We have

$$
\begin{aligned}
f\left(\sum_{j} x_{j} a_{j}\right) & \leqq \sum_{j} y_{j} u_{j} \\
& =\sum_{j}\left(y_{j} u_{j}^{\theta}\right) u_{j}^{1-\theta} \\
& \leqq\left(\sum_{j} y_{j}^{1 / \theta} u_{j}\right)^{\theta}\left(\sum_{j} u_{j}\right)^{1-\theta} \text { by Hölder's inequality. }
\end{aligned}
$$

Now $\sum_{j} y_{j}^{1 / \theta} u_{j}=\sum_{j} \varepsilon_{j} y_{j}^{1 / \theta} f\left(a_{j}\right) \leqq\left\|\sum_{j} \varepsilon_{j} y_{j}^{1 / \theta} a_{j}\right\|$, where $\varepsilon_{j} \in\{-1,1\}$. So by (ii'),

$$
\left(\sum_{j} y_{j}^{1 / \theta} u_{j}\right)^{p} \leqq \sum_{j} \lambda_{j} y_{j}^{y}
$$

and hence

$$
\left\|\sum_{j} x_{j} a_{j}\right\|^{r} \leqq \sum_{j} \lambda_{j} y_{j}^{\prime}\|T\|^{r-p}
$$

It follows, by the easy implication in Pietsch's theorem that

$$
\pi_{r}(T)^{r} \leqq \sum_{j} \lambda_{j}\|T\|^{r-p}=\pi_{p}(T)^{p}\|T\|^{r-p}
$$


Note. More generally, one can show that

$$
\pi_{r}(T) \leqq \pi_{p}(T)^{\theta} \pi_{q}(T)^{1-\theta}
$$

where $1 / r=\theta / p+(1-\theta) / q$. Simple examples show that these results do not hold for operators whose domain is not an $l_{\infty}$-space.

\section{Deduction of Grothendieck's inequality}

Take $T$ in $L\left(l_{\infty}^{m}, l_{1}^{p}\right)$. We have

$$
\begin{aligned}
\tilde{\pi}_{2}(T)^{2} & \leqq 2 \sqrt{3} \pi_{4}(T)^{2} & & \text { by Proposition } 2 \\
& \leqq 2 \sqrt{3} \pi_{2}(T)\|T\| & & \text { by Proposition } 3 \\
& \leqq 2 \sqrt{3} \tilde{\pi}_{2}(T)\|T\| & & \text { by Lemma } 1 .
\end{aligned}
$$

Hence $\tilde{\pi}_{2}(T) \leqq 2 \sqrt{3}\|T\|$. By Proposition 1 , this is equivalent to Grothendieck's inequality (with $K_{G} \leqq 2 \sqrt{3}$ ).

\section{Remarks}

(1) Clearly, there is a constant $K \leqq K_{G}$ such that $\pi_{2}(T) \leqq K\|T\|$ for all $T$ in $L\left(l_{\infty}^{m}, l_{1}^{p}\right)$. (The main part of [4] is concerned with generalizations of this result, rather than Grothendieck's inequality itself.) The exact value of $K$ remains unknown, and is of interest as much as $K_{G}$. It is well known that $K_{G} \leqq K \sqrt{\pi / 2}$ in the real case, and $K_{G} \leqq 2 K / \sqrt{\pi}$ in the complex case. It is attractive to conjecture that $K=\sqrt{2}$ in the real case, as it is elementary that this is the value for $l_{1}^{2}$. It is also the value for $l_{1}^{3}$, since $\pi_{2}(T)^{2} \leqq \pi_{1}(T)\|T\|$ and $\pi_{1}\left(l_{1}^{3}\right)=2$ (however, the estimate given by this reasoning grows with $p$ ).

(2) It is easy to show (as in Proposition 2, but without Khinchin's inequality) that $\tilde{\pi}_{2}(T) \leqq 3^{1 / 4} \tilde{\pi}_{4}(T)$. However, Proposition 3 becomes false if $\tilde{\pi}_{p}$, $\tilde{\pi}_{r}$ are substituted for $\pi_{p}, \pi_{r}$.

(3) Using the exact value of the corresponding Khinchin constant [1], one obtains a slightly better estimate using $\pi_{5}$ instead of $\pi_{4}: K_{G} \leqq 4 / \pi^{1 / 6}(=3.305$. .). This is the best estimate afforded by this method using $\pi_{p}$ for integral $p$.

Acknowledgement. This work was carried out while visiting the Technion, Haifa, Israel. I am indebted to Prof. Yehoram Gordon for some very helpful discussions.

\section{REFERENCES}

1. U. HAageruP, The best constants in the Khintchine inequality, Studia Math. 70 (1982), 231-283. 
2. J. L. Krivine, Théorèmes de factorisation dans les espaces réticulés, Séminaire MaureySchwartz 1973-74, exp. 22-23.

3. J. Lindenstrauss and L. Tzafriri, Classical Banach Spaces I (Springer, 1977).

4. G. PISIER, Grothendieck's theorem for noncommutative $C^{*}$-algebras, with an appendix on Grothendieck's constants, J. Funct. Anal. 29 (1978), 397-415.

Department of Mathematics

UNIVERSITY OF LANCASTER

LANCASTER

Great Britain 